\title{
Simultaneous, noninvasive, and transdermal extraction of urea and homocysteine by reverse iontophoresis
}

This article was published in the following Dove Press journal:

International Journal of Nanomedicine

19 February 201 I

Number of times this article has been viewed

\author{
Congo Tak-Shing Ching ${ }^{1,2,3}$ \\ Tzong-Ru Chou' \\ Tai-Ping Sun ${ }^{1,2}$ \\ Shiow-Yuan Huang ${ }^{3}$ \\ Hsiu-Li Shieh ${ }^{2}$ \\ 'Graduate Institute of Biomedicine \\ and Biomedical Technology; \\ ${ }^{2}$ Department of Electrical Engineering, \\ National Chi Nan University, Nantou, \\ Taiwan; ${ }^{3}$ Department of Photonics \\ and Communication Engineering, Asia \\ University, Taichung, Taiwan, Republic \\ of China
}

Background: Cardiovascular and kidney diseases are a global public health problem and impose a huge economic burden on health care services. Homocysteine, an amino acid, is associated with coronary heart disease, while urea is a harmful metabolic substance which can be used to reflect kidney function. Monitoring of these two substances is therefore very important. This in vitro study aimed to determine whether homocysteine is extractable transdermally and noninvasively, and whether homocysteine and urea can be extracted simultaneously by reverse iontophoresis.

Methods: A diffusion cell incorporated with porcine skin was used for all experiments with the application of a direct current (dc) and four different symmetrical biphasic direct currents (SBdc) for 12 minutes via $\mathrm{Ag} / \mathrm{AgCl}$ electrodes. The dc and the SBdc had a current density of $0.3 \mathrm{~mA} / \mathrm{cm}^{2}$.

Results: The SBdc has four different phase durations of $15 \mathrm{sec}, 30 \mathrm{sec}, 60 \mathrm{sec}$, and $180 \mathrm{sec}$. It was found that homocysteine can be transdermally extracted by reverse iontophoresis. Simultaneous extraction of homocysteine and urea by reverse iontophoresis is also possible.

Conclusion: These results suggest that extraction of homocysteine and urea by SBdc are phase duration-dependent, and the optimum mode for simultaneous homocysteine and urea extraction is the SBdc with the phase duration of $60 \mathrm{sec}$.

Keywords: reverse iontophoresis, homocysteine, urea, monitoring, noninvasive, transdermal

\section{Introduction}

Cardiovascular disease is a challenging global public health problem, causing about 16.7 million deaths annually worldwide. ${ }^{1}$ Meanwhile, kidney disease is reportedly increasing in developed countries, including the US, Canada, Australia, and Europe. 2,3 Cardiovascular disease and kidney disease impose a substantial economic burden, not just on health care services but also on industry and commerce. Taiwan also faces these challenges, with heart disease and kidney disease being, respectively, the second and eighth leading causes of death in $2007 .{ }^{4}$ Therefore, there is a need to reduce the occurrence and death rate by means of frequent monitoring of patients who are at high risk of developing cardiovascular disease or kidney disease. On the other hand, a large clinical study has shown that the increased risk of heart disease across the spectrum of kidney disease has been recently appreciated by the medical community. ${ }^{5,6}$ Therefore, simultaneous monitoring of cardiovascular disease and kidney disease is essential.

Homocysteine is an amino acid. Highly elevated homocysteine levels were associated with a more than three-fold increase in the risk of heart attack over a five-year period. ${ }^{5}$ Therefore, the blood homocysteine level is a good index for heart attack
Correspondence: Congo Tak-Shing Ching Graduate Institute of Biomedicine and Biomedical Technology, National Ch Nan University, No I University Rd, Puli, Nantou County 5456I, Taiwan, Republic of China

Tel +886 4929 I0960 ext 4774

Fax +886 492912434

Email tsching@ncnu.edu.tw 
monitoring. Conversely, urea in the plasma increases with the degree of renal failure and accumulates in kidney disease. ${ }^{6}$ Therefore, the clinical diagnosis of chronic kidney disease is principally based on the measurement of increased levels of urea.

Blood sampling is the current routine clinical method to determine blood homocysteine and urea levels. However, this method is invasive, painful, aesthetically unpleasant, and inconvenient. This provides considerable impetus for the development of noninvasive methods for the simultaneous monitoring of blood homocysteine and urea levels. So far, no research has been reported on investigation of transdermal extraction of homocysteine by reverse iontophoresis. Several papers have reported on the use of reverse iontophoresis to enhance the extraction of urea, ${ }^{7-9}$ but no papers have been found for the simultaneous transdermal extraction of urea and homocysteine by this method.

Reverse iontophoresis refers to the passage of a low voltage $(\leq 10 \mathrm{~V})$ and low level of current $\left(\leq 0.5 \mathrm{~mA} / \mathrm{cm}^{2}\right)$ through the skin to promote the extraction of both charged and neutral molecules. ${ }^{10}$ The most successful reverse iontophoresis application for patient monitoring to date, as mentioned, has been in noninvasive and continuous blood glucose detection utilizing the GlucoWatch ${ }^{\circledR} .{ }^{11,12}$

The aim of this study was to find out whether homocysteine is extractable transdermally and noninvasively, and whether homocysteine and urea can be extracted simultaneously by reverse iontophoresis.

\section{Materials and methods}

\section{Reagents and solutions}

All reagents used in this study were commercially available and used without further purification. Phosphate-buffered saline, urea, and homocysteine were purchased from Sigma Chemical Company (St Louis, MO). The urea assay kit was purchased from BioVision (Mountain View, CA). The homocysteine assay kit was purchased from IBL-America (Minneapolis, MN). Deionized water, purified by a Millipore Milli-Q UFplus System (Bedford, MA) was used to prepare all solutions.

\section{Equipment}

The diffusion cell used for the in vitro studies was the same as that previously described (Figure 1 ). ${ }^{13}$ A reverse iontophoresis device was homemade, with an accuracy of $\pm 0.01 \mu \mathrm{A}$ for delivering current and $\pm 0.01 \mathrm{msec}$ for timing. All colorimetric analysis was performed using a Bio-Rad 680 microplate reader (Bio-Rad, Hemel Hempstead, UK).

\section{In vitro studies}

The diffusion cell was used for all experiments. It consisted of two upper electrode chambers and a lower chamber. The electrode chambers were separated from the lower chamber by porcine ear skin with a thickness of $250 \mu \mathrm{m}$, obtained by Padgett Dermatome (Integra LifeSciences Corporation, Plainsboro, NJ). The thickness of the skin used in this study was the same as that reported in other researchers'

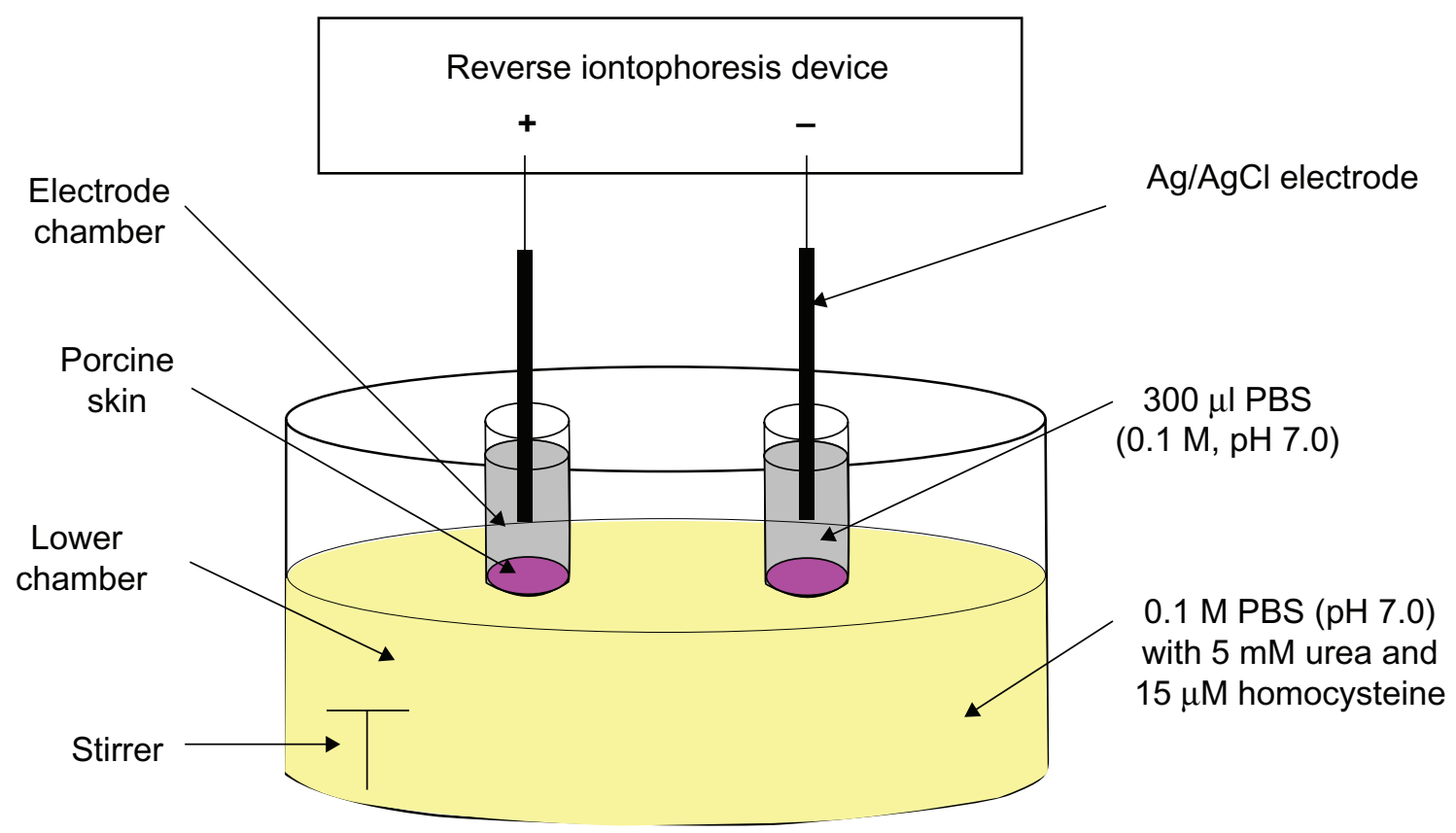

Figure I Diffusion cell for all in vitro experiments. 
diffusion studies. ${ }^{17,18}$ All chambers were filled with $0.1 \mathrm{M}$ phosphate buffer ( $\mathrm{pH} 7.0$ ), except that the lower chamber additionally contained $5 \mathrm{mM}$ urea and $15 \mu \mathrm{M}$ homocysteine, which attempted to simulate the normal physiological levels of urea and homocysteine in human blood. Each upper electrode chamber contained $300 \mu \mathrm{L}$ phosphate buffer $(0.1 \mathrm{M}$, $\mathrm{pH}$ 7.0) while the lower chamber contained the necessary solution of about $25 \mathrm{~mL}$. A Ag/ $\mathrm{AgCl}$ electrode was positioned inside each electrode chamber, and its fabrication procedure was the same as previously described. ${ }^{13}$ The surface area of the porcine skin exposed to the electrode in each chamber was $0.2 \mathrm{~cm}^{2}$ and the electrode chambers were $11 \mathrm{~mm}$ apart.

A direct current (dc, cathodal dc abbreviated to dc-cathode and anodal dc abbreviated to dc-anode) and four symmetrical biphasic direct currents (SBdc) were applied for 12 minutes and 36 minutes via the $\mathrm{Ag} / \mathrm{AgCl}$ electrodes. The dc and the $\mathrm{SBdc}$ had a current density of $0.3 \mathrm{~mA} / \mathrm{cm}^{2}$. The four SBdc had four different phase durations of $15 \mathrm{sec}$ (15s PDSBdc), $30 \mathrm{sec}$ (30s PDSBdc), $60 \mathrm{sec}$ (60s PDSBdc), and $180 \mathrm{sec}$ (180s PDSBdc). At the end of each experiment, the entire contents of the electrode chambers were removed to determine the amount of urea and homocysteine extracted.

For the control experiments, all the experimental arrangements and procedures were the same, except no current was applied.

\section{Determination of extracted urea and homocysteine}

The amount of urea (determined by the urea assay kit) and homocysteine (determined by the homocysteine assay kit) extracted through the porcine skin were quantified by a colorimetric clinical chemistry assay method adapted for use with the Bio-Rad 680 microplate reader. To perform the urea and homocysteine quantifications, we followed their corresponding assay procedures mentioned in the user manuals of their assay kits, ${ }^{19,20}$ except that the amount of sample used was $100 \mu \mathrm{L}$ rather than the recommended amount. An excellent linear relationship $\left(r^{2}>0.95\right)$ between urea concentration and its relative absorbance was found, and the relationship between homocysteine concentration and its relative absorbance was also found to be linearly excellent $\left(r^{2}>0.9\right)$. This allowed the urea and homocysteine concentrations to be calculated simply by linear regression.

\section{Statistical analysis}

One-way analysis of variance was used to determine whether there were significant differences between the dc and SBdc for urea and homocysteine extraction. Post hoc comparisons were made with the least significant difference (LSD) test. All statistical analyses were carried out using SPSS 10.0 software (SPSS, Chicago, IL), with the level of statistical significance set at $P<0.05$.

\section{Results and discussion}

An in vitro model was used in this study because of its simplicity. We were able to create a normal (ie, healthy) model, a cardiovascular disease model, and a kidney disease model, by just simply adjusting the solution stored inside the lower chamber of the diffusion cell. For example, the kidney disease model could be created by preparing the lower chamber solution with a high urea concentration, ie, $20 \mathrm{mM}$ urea. On the other hand, it is relatively difficult and expensive to create an in vivo model. Hence, an in vitro model was used in this study. We simply used the normal model ( $5 \mathrm{mM}$ urea and $15 \mu \mathrm{M}$ homocysteine) for this investigation in order to find out whether homocysteine was extractable, as well as whether homocysteine and urea could be extracted simultaneously. In a future study, cardiovascular disease and kidney disease models will be used.

Skin consists of three layers, ie, the epidermis, dermis, and subcutis. The epidermis (about $100 \mu \mathrm{m}$ in thickness) is the topmost layer of the skin, forming a barrier for many substances going in and out. The in vitro model used in this study also had these three layers. The diffusion cell used in this study had a lower chamber, and its function was to simulate the dermis and subcutis. For the epidermis, a porcine ear skin was used, and its thickness $(250 \mu \mathrm{m})$ was the same as the thickness reported in diffusion studies done by other researchers. ${ }^{17,18}$

There are three mechanisms for reverse iontophoresis, ie, diffusion (almost negligible), electromigration, and electro-osmosis. The major mechanisms for transport of ions and molecules are electromigration of charged species to the electrode of opposite polarity, electro-osmosis of neutral molecules to the cathode or anode, or a combination of these two processes.

Homocysteine, an uncharged molecule with a molecular weight of about $135 \mathrm{Da}$, is a hydrophilic molecule (partition coefficient -2.2). Because of its small size and hydrophilic properties, homocysteine is expected to be transdermally extractable by electro-osmosis. Urea is a negatively charged molecule and its molecular weight is about 60.06 Da. As a consequence of its small size and negative charge, electromigration is the dominant physical factor for urea extraction.

As expected, reverse iontophoresis facilitated the transdermal extraction of homocysteine and urea (see Figure 2). 


\section{A)}

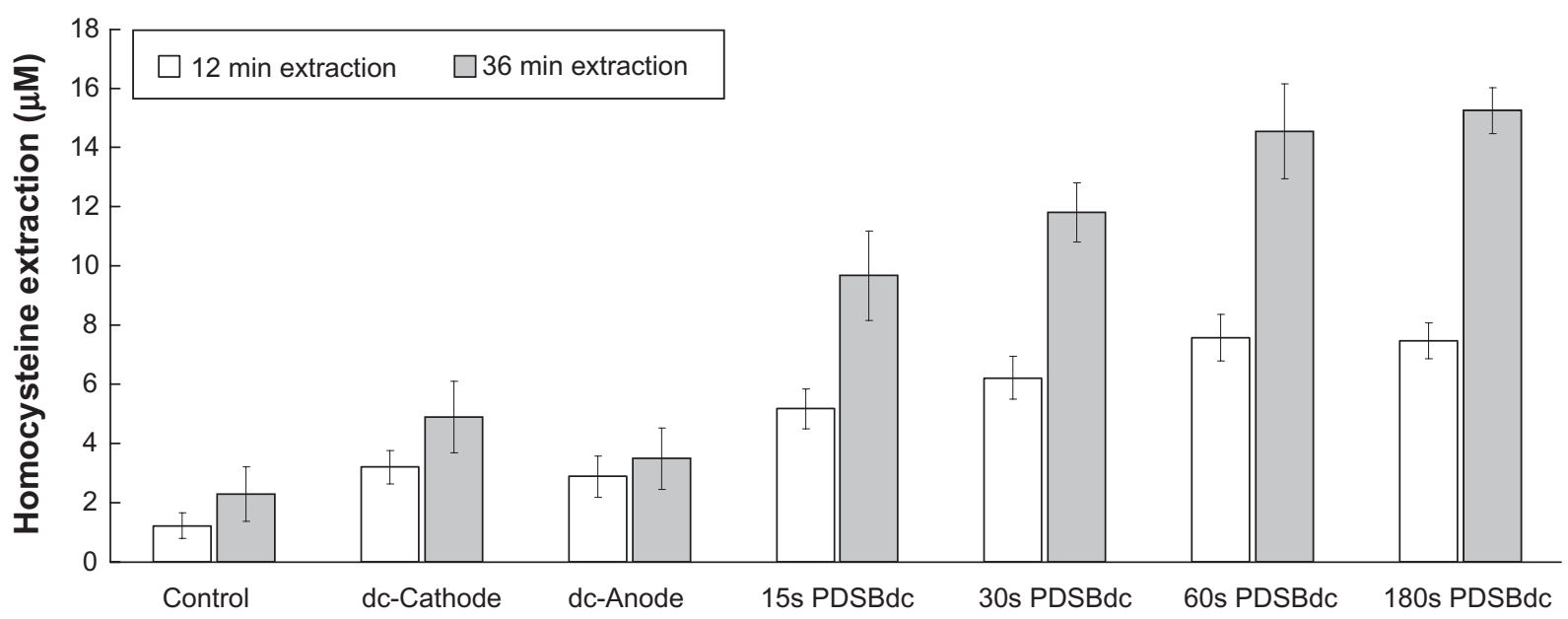

B)

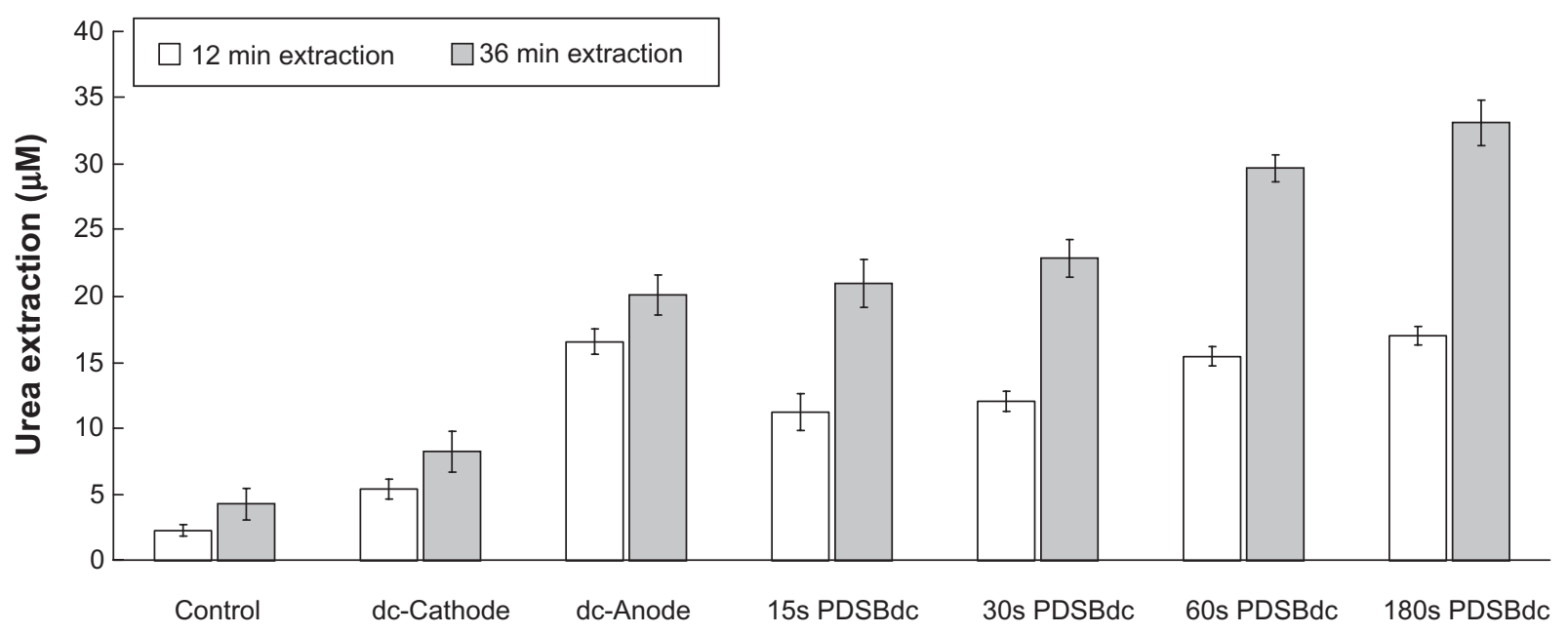

Figure 2 The transdermal extraction of $\mathbf{A}$ ) homocysteine and $\mathbf{B}$ ) urea (mean \pm standard deviation; $\mathrm{n} \geq 3$ for each bar) by reverse iontophoresis. The extraction period was 12 minutes and 36 minutes. The current density of the extraction was $0.3 \mathrm{~mA} / \mathrm{cm}^{2} ; 15 \mathrm{~s}, 30 \mathrm{~s}, 60 \mathrm{~s}$, and $180 \mathrm{~s}$ PDSBdc corresponds to the symmetrical biphasic direct current with the phase duration of 15 seconds, 30 seconds, 60 seconds, and 180 seconds, respectively. The dc-cathode and dc-anode correspond to a cathode of direct current and an anode of direct current, respectively.

Figure 2A shows that there was a significant difference between the dc and the four SBdc on extraction of homocysteine (one-way analysis of variance, $P<0.001$ ). It was found that there was a significantly higher homocysteine extraction for the dc and the four SBdc than for the control sample which utilized diffusion-only extraction (LSD test, $P<0.001$ in most cases and $P<0.05$ in the remaining cases). This suggests that the use of physical force, like reverse iontophoresis, can promote more homocysteine extraction than diffusion alone. Because homocysteine is an uncharged molecule, it theoretically should be extracted to the electrode chamber of the diffusion cell with the negative polarity (ie, the cathode). As shown in Figure 2A, although slightly higher homocysteine extraction was observed at the cathode than at the anode, the difference was not significant. This might be because prolonged dc passage can cause skin polarization, which results in the reduction of electro-osmotic flow. Homocysteine extractions at the dc-cathode and the dc-anode were found to be significantly lower than that at the four SBdc (LSD test, $P<0.001$ in most cases and $P<0.05$ in the remaining cases). A possible explanation is that the use of dc for reverse iontophoresis causes skin polarization. Polarization effects turn the skin into what is effectively a charged capacitor, and this reduces the flow of current and charged species during reverse iontophoresis. However, the use of SBdc has been suggested as a means to 
overcome skin polarization. ${ }^{14,15}$ Therefore, SBdc promoted more homocysteine extraction than dc. Also, homocysteine extraction using 60s PDSBdc was found to be significantly higher than that with protocols utilizing 15s PDSBdc (LSD test, $P<0.001$ ) and 30s PDSBdc (LSD test, $P<0.05$ ) but there was no significant difference when using 180s PDSBdc (LSD test, $P=0.838$ ). This result suggests that extraction of homocysteine by SBdc is phase duration-dependent. Because a plateau was observed at 60s PDSBdc and 180s PDSBdc for homocysteine extraction, the optimum mode for homocysteine extraction is therefore the $60 \mathrm{~s}$ SBdc. Moreover, it was found that a longer extraction time resulted in relatively more homocysteine extraction. However, a three-fold increase in extraction time did not result in a three-fold increase in homocysteine extraction (see Figure 2A). More importantly, the homocysteine extraction flux (see Figure 3A) and even effective homocysteine extraction (see Table 1) decreased with increased extraction time. This indicates the presence of a homocysteine reservoir in the skin.

On the other hand, as shown in Figure 2B, a significant difference was found between dc and the four SBdc for the extraction of urea (one-way analysis of variance, $P<0.001$ ). Again, the dc and the four SBdc were found to promote more urea extraction than diffusion alone (LSD test, $P<0.001$ in most cases and $P<0.05$ in the remaining cases). Because urea carries a negative charge, urea should theoretically be extracted to the electrode chamber of the diffusion cell with the positive polarity. As shown in Figure 2B, our results agreed with this theoretical phenomenon that the dc-anode facilitates significantly $(P<0.001)$ more urea extraction than the dc-cathode. Urea extraction using the dc-anode was found to be significantly higher than that of protocols utilizing

A)

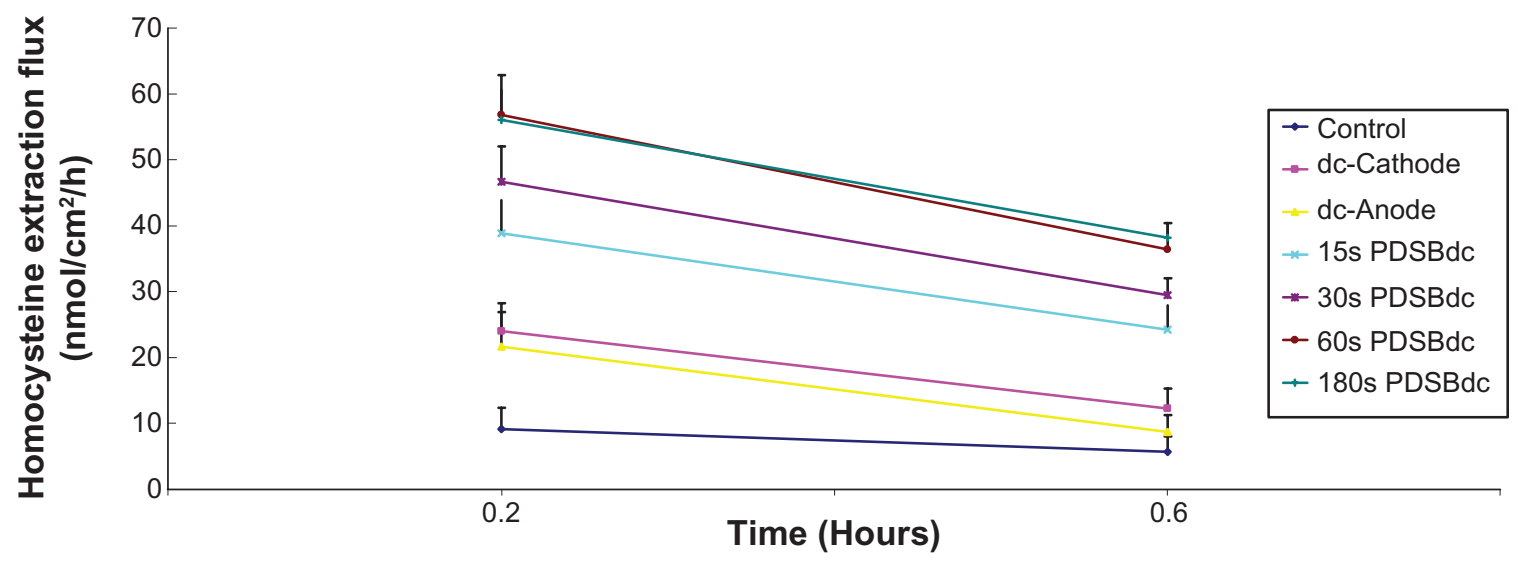

B)

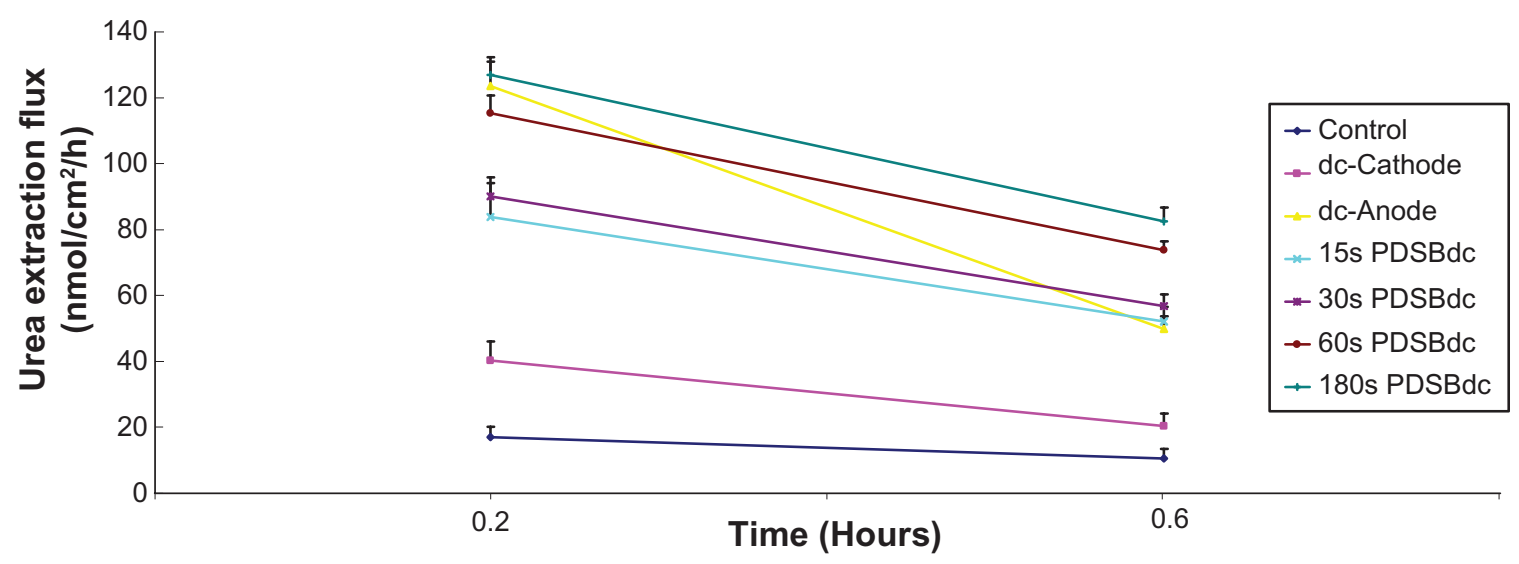

Figure 3 A) Homocysteine and B) urea extraction fluxes as a function of time (mean \pm standard deviation; $\mathrm{n} \geq 3$ ). The current density of the extraction was $0.3 \mathrm{~mA} / \mathrm{cm}^{2}$; $15 \mathrm{~s}, 30 \mathrm{~s}, 60 \mathrm{~s}$, and 180s. PDSBdc corresponds to the symmetrical biphasic direct current with the phase duration of 15 seconds, 30 seconds, 60 seconds, and I80 seconds, respectively. The dc-cathode and dc-anode corresponds to a cathode of direct current and an anode of direct current, respectively. 
Table I Effect of the direct current and the symmetrical biphasic direct currents on effective urea and homocysteine extraction $(n \geq 3)$

\begin{tabular}{|c|c|c|c|c|}
\hline $\begin{array}{l}\text { Current } \\
\text { waveform }\end{array}$ & $\begin{array}{l}\text { Experimental } \\
\text { time (h) }\end{array}$ & $\begin{array}{l}\text { Effective extraction } \\
\text { time }^{\text {a }}(\mathrm{h})\end{array}$ & $\begin{array}{l}\text { Effective urea } \\
\text { extraction }{ }^{\text {b }}(\mathrm{nmol} / \mathrm{h})\end{array}$ & $\begin{array}{l}\text { Effective homocysteine } \\
\text { extractionc (nmol/h) }\end{array}$ \\
\hline Control & 0.2 & 0.2 & $3.4 \pm 0.7$ & $1.8 \pm 0.6$ \\
\hline dc-cathode & 0.2 & 0.2 & $8.1 \pm 1.2$ & $4.8 \pm 0.9$ \\
\hline dc-anode & 0.2 & 0.2 & $24.8 \pm 1.5$ & $4.3 \pm 1.1$ \\
\hline I5s PDSBdc & 0.2 & 0.1 & $33.6 \pm 4.2$ & $15.5 \pm 2.0$ \\
\hline 30s PDSBdc & 0.2 & 0.1 & $36.1 \pm 2.3$ & $18.7 \pm 2.1$ \\
\hline 60s PDSBdc & 0.2 & 0.1 & $46.3 \pm 2.1$ & $22.7 \pm 2.4$ \\
\hline 180s PDSBdc & 0.2 & 0.1 & $50.9 \pm 2.1$ & $22.4 \pm 1.8$ \\
\hline Control & 0.6 & 0.6 & $2.1 \pm 0.6$ & $\mathrm{I} . \mathrm{I} \pm 0.5$ \\
\hline dc-cathode & 0.6 & 0.6 & $4.1 \pm 0.8$ & $2.5 \pm 0.6$ \\
\hline dc-anode & 0.6 & 0.6 & $10.0 \pm 0.8$ & $1.7 \pm 0.5$ \\
\hline I5s PDSBdc & 0.6 & 0.3 & $20.9 \pm 1.8$ & $9.7 \pm 1.5$ \\
\hline 30s PDSBdc & 0.6 & 0.3 & $22.8 \pm 1.4$ & $11.8 \pm 1.0$ \\
\hline 60s PDSBdc & 0.6 & 0.3 & $29.6 \pm 1.0$ & $14.5 \pm 1.6$ \\
\hline I80s PDSBdc & 0.6 & 0.3 & $22.1 \pm 1.7$ & $15.3 \pm 1.8$ \\
\hline
\end{tabular}

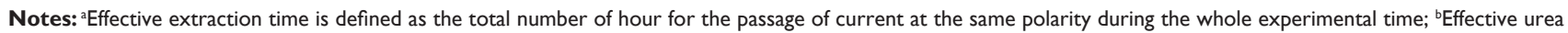
extraction $=$ Number of mole of extracted urea after the whole experiment/Effective extraction time; ${ }^{\mathrm{C} E f f e c t i v e ~ h o m o c y s t e i n e ~ e x t r a c t i o n ~}=$ Number of mole of extracted homocysteine after the whole experiment/Effective extraction time.

15s PDSBdc and 30s PDSBdc (LSD test, $P<0.001$ in all cases) but there was no significant difference using $60 \mathrm{~s}$ PDSBdc (LSD test, $P=0.139$ ) and 180s PDSBdc (LSD test, $P=0.540)$. However, as shown in Table 1, it could be argued that because the dc and SBdc have different effective extraction times (defined as the total number of hours for the passage of current at the same polarity during the whole experimental time), a standardization technique should be employed to investigate the rate of transdermal extraction of urea. Therefore, the extraction rate for urea was recalculated in nmol/hour, where time was set as the actual time of the applied current at the electrode chamber collecting the molecule. This is based on the assumption that, at any time in the cycle, the anode dominates the collection of urea. As shown in Table 1, calculating the effective urea extraction amount in this format shows that urea extraction using the dc-anode was significantly lower than when using SBdc (LSD test, $P<0.001$ in all cases). On the other hand, urea extraction using 60s PDSBdc was found to be significantly higher than when using 15s PDSBdc and 30s PDSBdc (LSD test, $P<0.001$ in all cases) but lower than that when using 180 s PDSBdc (LSD test, $P<0.05$ ). This result suggests that extraction of urea by SBdc is also phase duration-dependent. Although 15s, 30s, 60s, and 180s PDSBdc showed the same effective urea extraction time, it was found that a long PDSBdc results in higher urea extraction. Because skin has a thickness dimension, urea takes some time to pass through the skin. For a short PDSBdc, some urea molecules may not have enough time to pass through and may stay inside the skin. These urea molecules will not move forward into the collection chamber, and may even move backwards to the lower chamber of the diffusion cell when the phase of the SBdc is reversed. This might explain the phase duration-dependent phenomenon. Because urea extraction using 60s PDSBdc was slightly lower than that using 180s PDSBdc (LSD test, $P=0.045$ ), the optimum mode for urea extraction is $60 \mathrm{~s}$ SBdc in order to achieve simultaneous extraction of urea and homocysteine. On the other hand, it was found that a longer extraction time caused comparatively more urea extraction, although a threefold increase in extraction time could not cause a three-fold increase in urea extraction (see Figure 2B). Moreover, the urea extraction flux (see Figure 3B) and even the effective urea extraction (see Table 1) decreased with increased extraction time. This also indicates the presence of a urea reservoir in the skin. Conversely, as shown in Figure 3B, the result of dcanode urea extraction flux in 36 minutes (about $0.05 \mu$ mole/ $\mathrm{cm}^{2} /$ hour $\approx 0.01 \mu$ mole/hour) was found to be in agreement with the findings reported by Wascotte et al in 2007. ${ }^{7}$ They found that the urea extraction flux in 30 minutes was about $0.015 \mu \mathrm{mole} /$ hour. However, our finding of PDSBdc urea extraction flux in 36 minutes (see Figure 3B) was higher than that of Wascotte et al. This means that PDSBdc resulted in a higher urea extraction flux as compared with dc alone.

Based on our findings, reverse iontophoresis potentially provides a promising technique for simultaneous, noninvasive, and transdermal extraction of urea and homocysteine. However, it does not mean that this is applicable in humans. Therefore, ethic approval has been applied for to do further 
research. Once the human study is approved, experiments will be conducted to test our hypothesis that homocysteine is transdermally extractable, and that homocysteine and urea can be simultaneously extracted in humans. If our hypothesis is correct, a homocysteine-urea Glucowatch may be able to be developed in the future. This could help potential users to have a screening test inhouse. Of course, a homocysteine-urea watch may face the same reproducibility problems as those found with the Glucowatch. Therefore, intensive research will be necessary to overcome this reproducibility problem.

\section{Conclusion}

A novel method for noninvasive transdermal extraction of homocysteine by reverse iontophoresis has been demonstrated. Reverse iontophoresis facilitates transdermal extraction of homocysteine and urea at the same time. The optimum mode for simultaneous homocysteine and urea extraction appears to be SBdc with a phase duration of 60 seconds. Once this hypothesist is proven in humans in the future, this new method for urea and homocysteine monitoring would be beneficial to patients, such as those with kidney disease, or obese individuals who are at high risk of developing coronary artery disease.

\section{Acknowledgments}

This work was supported by a grant (98-2221-E-260-002-) from the National Science Council, Taiwan, Republic of China. It was also partially supported by a grant (99T8888-6) from the National Chi Nan University, Taiwan, Republic of China, and another grant (98-2221-E-260-024-MY3) from the National Science Council, Taiwan, Republic of China.

\section{Disclosure}

The authors report no conflicts of interest in this work.

\section{References}

1. WHO. Cardiovascular Disease Prevention and Control, 2003. Available at: http://www.who.int. Accessed November 17, 2010.

2. Goeree R, Manalich J, Grootendorst P, Beecroft ML, Churchill DN. Cost analysis of dialysis treatments for end-stage renal diseases. Clin Invest Med. 1996;18:455-464.
3. Raine AEG, Margreiter R, Brunner FP. Report on management of renal failure in Europe, XXII, 1991. Nephrol Dial Transplant. 1992;71 Supp1 2: $7-35$.

4. Department of Health, Executive Yuan. Health statistical reports, 2008. Available at: http://www.doh.gov.tw. Accessed November 17, 2010.

5. Stampfer MJ, Malinow MR, Willett WC, et al. A prospective study of plasma homocyst(e)ine and risk of myocardial infarction in US physicians. JAMA. 1992;268:877-881.

6. Man NK, Touam M, Jungers P. Causes et consequences de l'uremie chronique, indications de la dialyse de suppleance. In: L'Hemodialyse de Suppleance. Flammarion, Paris: Medecine-Sciences; 2003.

7. Wascotte V, Rozet E, Salvaterra A, et al. Non-invasive diagnosis and monitoring of chronic kidney disease by reverse iontophoresis of urea in vivo. Eur J Pharm Biopharm. 2008;69:1077-1082.

8. Wascotte V, Delgado-Charro MB, Rozet E, et al. Monitoring of urea and potassium by reverse iontophoresis in vitro. Pharm Res. 2007;24:1131-1137.

9. Degim IT, Ilbasmis S, Dundaroz R, et al. Reverse iontophoresis: A noninvasive technique for measuring blood urea level. Pediatr Nephrol. 2003;18:1032-1037.

10. Merino V, Kalia YN, Guy RH. Transdermal therapy and diagnosis by iontophoresis. Trends Biotechnol. 1997;15:288-290.

11. Pitzer KR, Desai S, Dunn T, et al. Detection of hypoglycaemia with the GlucoWatch biographer. Diabetes Care. 2001;24:881-885.

12. Tierney MJ, Tamada JA, Potts RO, et al. Clinical evaluation of the GlucoWatch ${ }^{\circledR}$ biographer: A continual, non-invasive glucose monitor for patients with diabetes. Biosens Bioelectron. 2001;16:621-629.

13. Ching TS, Connolly P. Simultaneous transdermal extraction of glucose and lactate from human subjects by reverse iontophoresis. Int J Nanomedicine. 2008;3:211-223.

14. Howard JP, Drake TR, Kellogg DL. Effects of alternating current iontophoresis on drug delivery. Arch Phys Med Rehabil. 1995;76: 463-466.

15. Tomohira Y, Machida Y, Onishi H, et al. Iontophoretic transdermal absorption of insulin and calcitonin in rats with newly-devised switching technique and addition of urea. Int J Pharm. 1997;155:231-239.

16. Mann JF, Gerstein HC, Pogue J, et al. Renal insufficiency as a predictor of cardiovascular outcomes and the impact of ramipril: The HOPE randomized trial. Ann Intern Med. 2001;134:629-636.

17. Chen J, Hammell DC, Spry M, et al. In vitro skin diffusion study of pure forskolin versus a forskolin-containing Plectranthus barbatus root extract. J Nat Prod. 2009;72:769-771.

18. Baynes RE, Brooks JD, Mumtaz M, et al. Effect of chemical interactions in pentachlorophenol mixtures on skin and membrane transport. Toxicol Sci. 2002;69:295-305.

19. Homocysteine enzymatic assay kit. Available at: http://www. ibl-america.com/pdf/newIBLelisa/IB46101.pdf. Accessed November 17, 2010.

20. Urea assay kit. Available at: http://www.biovision.com/manuals/K375100.pdf?osCsid=j6kit0eqdved7ebtnsjv5g0010. Accessed November 17, 2010 .
International Journal of Nanomedicine

\section{Publish your work in this journal}

The International Journal of Nanomedicine is an international, peerreviewed journal focusing on the application of nanotechnology in diagnostics, therapeutics, and drug delivery systems throughout the biomedical field. This journal is indexed on PubMed Central, MedLine, CAS, SciSearch $\AA$, Current Contents ${ }^{\circledR} /$ Clinical Medicine,

\section{Dovepress}

Journal Citation Reports/Science Edition, EMBase, Scopus and the Elsevier Bibliographic databases. The manuscript management system is completely online and includes a very quick and fair peer-review system, which is all easy to use. Visit http://www.dovepress.com/ testimonials.php to read real quotes from published authors. 